\title{
EOPs: Ácido ursodeoxicólico para la prevención de colelitiasis en obesos con descenso rápido de peso
}

Ursodeoxycholic acid for cholelithiasis in obese subjects with rapid weight reduction

Traducido con autorización del National Institute for Health and Clinical Excellence (NICE). Disponible en: http://www.nice.org.uk/nicemedia/live/12327/45865/45865.pdf

\section{Resumen}

Se evaluó la evidencia para determinar si el tratamiento profiláctico con ácido ursodeoxicólico es seguro y eficaz para prevenir la formación de litiasis vesicular en pacientes obesos que participan en un programa para bajar de peso y/o en quienes son sometidos a cirugía bariátrica. De acuerdo con los consistentes resultados entre los estudios encontrados, estaría recomendado el uso de ácido ursodeoxicólico en la población y escenario previamente descriptos.

\section{Abstract}

We evaluated the evidence to determine whether prophylactic treatment with ursodeoxycholic acid is safe and effective to prevent the formation of gallstones in obese patients participating in a weight loss program or who received bariatric surgery. According to the consistent results between the studies found, ursodeoxycholic acid would be recommended in the population and scenario previously described.

Palabras clave: colelitiasis, obesidad, ácido ursodesoxicólico, pérdida de peso, cirugía bariátrica.. Key words: cholelithiasis, obesity, ursodeoxycholic acid, weight loss, bariatric surgery.

Mel MS. Ácido ursodeoxicólico para la prevención de colelitiasis en obesos con descenso rápido de peso. Evid. Act Práct Ambul Jul-Set 2011;14(3):118-119.

\section{Escenario Clínico}

Un médico de familia atendió a una mujer obesa de 56 años con antecedentes de melanoma, operado en el 2005, y cáncer de mama, resecado en el año 2007 , por los cuales perdió $14 \mathrm{~kg}$ de peso, que concurre a la consulta con el resultado de una ecografía abdominal. La misma había sido solicitada por su oncólogo al referirle un dolor nuevo en hipocondrio derecho relacionado con la ingesta de comidas grasas. El informe del estudio reveló la presencia de un lito de $7 \mathrm{~mm}$. en el interior de la vesicula biliar, no visible en una ecografía abdominal realizada un año atrás.

\section{Pregunta que generó el caso}

¿En pacientes obesos, que realizan un descenso de peso de manera rápida (población), ya sea por dietas hipocalóricas o cirugía de by pass gástrico, el uso de ácido ursodeoxicólico (intervención), disminuye el riesgo de litiasis vesicular (resultado)?

\section{Estrategia de búsqueda}

Se realizó una búsqueda en Up to date, y en la sección Clinical Queries de PubMed utilizando como palabras clave "lithiasis" y "obesity". De los resultados obtenidos, se seleccionaron tres ensayos clínicos aleatorizados y un meta-análisis.

\section{Litiasis vesicular en obesos que pierden peso rápidamente La litiasis vesicular es una de las enfermedades más comunes y costosas de todo el aparato digestivo. La ecografía juega un papel importante en la detección del proceso proveyendo un método diagnóstico rápido, y de bajo riesgo'. El rápido descenso de peso ya sea por una dieta hipocalórica o luego de una cirugía para el tratamiento de la obesidad mórbida está asociado a un aumento en la aparición de cálculos vesiculares. Se cree que la incidencia aproximada de litiasis vesicular dentro de este grupo es de $30 \%$. El mecanismo no es muy conocido, pero se cree que es porque disminuye la cantidad de ácidos biliares de la bilis y se enlentece el vaciamiento vesicular. En contraste con lo que ocurre en la población general, las personas que presentan colelitiasis debido a la pérdida de peso, presentan más síntomas ${ }^{2,3}$. El uso de estati- nas, así como también factores dietarios, como el consumo mo- derado de café y dietas balanceadas ricas en proteínas de origen vegetal, podrían ser protectores en cuanto a la formación de cál- culos en la vesícula.}

\section{Resumen de la evidencia}

Broomfield, PH y col. Effects of ursodeoxycholic acid and aspirin on the formation of lithogenic bile and gallstones during loss of weight. N Engl J Med 1988;319(24):1567-72.

Objetivo: determinar si la administración de aspirina o ácido ursodeoxicólico durante el descenso de peso pueden prevenir la aparición de formaciones litiásicas en el líquido biliar.
Diseño: ensayo clínico aleatorizado, doble ciego, controlado con placebo.

Población: participaron 68 pacientes obesos sin evidencia de litiasis vesicular que se encontraban en un programa de descenso de peso (520 Kcal/día).

Intervención: se asignó de forma aleatorizada el uso de ácido ursodeoxicólico (1200 mg/día), aspirina (1300 mg/día) o placebo por 16 semanas. Se realizó una ecografía a las cuatro semanas de tratamiento y a las tres semanas postratamiento para detectar la presencia de litiasis vesicular o duodenal, estudiar el líquido biliar para detectar cristales de colesterol y para determinar la saturación biliar y la concentración de glicoproteínas.

Resultados: No se detectó litiasis vesicular ni formación de cristales de colesterol en los pacientes tratados con ácido ursodeoxicólico, mientras que en los pacientes tratados con placebo, cinco presentaron litiasis vesicular $(p<0,05)$ y seis cristales de colesterol $(P<0.001)$.

De los que recibieron aspirina, dos formaron litiasis vesicular y un paciente cristales de colesterol (sin diferencias estadísticamente significativas en cuanto al uso de ácido ursodeoxicólico).

Para la semana cuatro, el índice de saturación biliar se incrementó en el grupo placebo $(\mathrm{P}<0.001)$, disminuyó en el grupo que recibió ácido ursodeoxicólico $(\mathrm{P}<0.001)$, y no cambió significativamente en el grupo que recibió aspirina.

La concentración de glicoproteínas en bilis se incrementó en los que recibieron placebo $(\mathrm{P}<0.001)$ pero no varió significativamente en los grupos tratados con ácido ursodeoxicólico o aspirina.

Conclusión: el ácido ursodeoxicólico previene la litiasis vesicular y los cambios litogénicos en la bilis de sujetos obesos que descienden de peso.

Shiffman ML, y col. Prophylaxis against gallstone formation with ursodeoxycholic acid in patients in very-low-calorie diet program. Ann Intern Med 1995;(12):899-905.

Objetivo: determinar si el tratamiento profiláctico con ácido ursodeoxicólico puede prevenir la formación de litiasis vesicular en personas que participan en un programa para bajar de peso con una dieta muy baja en calorías.

Diseño: ensayo clínico aleatorizado, multicéntrico, doble ciego, controlado con placebo. Los pacientes fueron tratados con placebo o con 300,600, ó 1200 mg/día de ácido ursodeoxicólico.

Población: 1.004 pacientes bajo una dieta de $520 \mathrm{Kcal} /$ día a base de líquidos y proteínas durante 16 semanas. Todos los 
pacientes tenían un índice de masa corporal (IMC) $\geq 38 \mathrm{~kg} / \mathrm{m}^{2}$ y una ecografía anterior al estudio con una vesícula normal. El IMC y el peso fueron medidos antes de comenzar la dieta y cada dos semanas hasta la semana 16 . Se realizó una ecografía de la vesícula al comenzar, a la semana 8 y a la semana 16 de comenzada la dieta.

Resultados: El IMC inicial promedio era de $44,2 \mathrm{~kg} / \mathrm{m} 2$. Se desarrolló litiasis vesicular en el $28 \%$ de los pacientes que recibían placebo (IC95\% 22 a 35), 8\% (5 a 13) de los pacientes tratados con $300 \mathrm{mg} /$ día de ácido ursodeoxicólico, 3\% (1 a 7) de los paciente tratados con $600 \mathrm{mg} /$ día de ácido ursodeoxicólico, y $2 \%$ (0,5 a 5) de los pacientes tratados con $1.200 \mathrm{mg} / \mathrm{día}$ de ácido ursodeoxicólico. La diferencia entre los pacientes que recibieron placebo y los que recibieron ácido ursodeoxicólico fue estadísticamente significativa y se observó un efecto dosis-respuesta.

Conclusión: el ácido ursodeoxicólico a dosis de $600 \mathrm{mg} / \mathrm{día}$, es altamente efectivo en la prevención de la litiasis vesicular en pacientes bajo una dieta diaria para disminuir de peso.

Miller K, y col. Gallstone Formation Prophylaxis After Gastric Restrictive Procedures for Weight Loss. Ann Surg. 2003;238(5):697-702.

Objetivo: determinar si el uso de ácido ursodeoxicólico administrado durante seis meses de manera profiláctica, es efectivo para prevenir la formación de litiasis vesicular.

Diseño: ensayo clínico aleatorizado, doble ciego, controlado con placebo.

Pacientes: pacientes con obesidad mórbida sometidos a bypass gástrico y banding gástrico ajustable.

Intervención: $500 \mathrm{mg} / \mathrm{día}$ de ácido ursodeoxicólico versus placebo, comenzando tres días antes de la cirugía y continuando hasta seis meses después o hasta que desarrollen litiasis vesicular. Se obtuvo una ecografía abdominal o una TC abdominal antes de la cirugía y a los tres, seis, 12 y 24 meses después de la cirugía o hasta que se desarrolló litiasis vesicular.

Resultados: de los 152 pacientes participantes, 76 fueron aleatorizados a placebo y 76 a $500 \mathrm{mg} /$ día de ácido ursodeoxicólico. El peso y el índice de masa corporal preoperatorio no fueron significativamente diferentes entre los dos grupos. La formación de litiasis vesicular fue significativamente menos frecuente con el uso de ácido ursodeoxicólico que con el placebo, tanto a los 12 meses ( $3 \%$ vs $22 \%$; $P=0,0018$ ) como a los 24 meses ( $8 \%$ versus $30 \%$; $\mathrm{P}=0,0022)$. Se realizó colecistectomía en el $4,7 \%$ de los pacientes del grupo intervención versus el $12 \%$ del grupo placebo $(P<0,02)$.

Conclusión: 500mg/día de ácido ursodeoxicólico durante seis meses es una profilaxis efectiva para prevenir el desarrollo de litiasis vesicular en procedimientos de restricción gástrica.

Uy MC, y col. Ursodeoxycholic acid in the prevention of gallstone formation after bariatric surgery: a meta-analysis. Obesity Surgery.2008:18(12):1532-8.
Objetivos: determinar la eficacia y seguridad del ácido ursodeoxicólico en la prevención de cálculos biliares después de la cirugía bariátrica.

Fuente de Datos: MEDLINE, EMBASE, CENTRAL, LILACS, Australasian Medical Index and Health Research and Development Information Network sin restricciones de idioma hasta septiembre de 2007. Se controlaron listas de referencias, y se contactaron autores y compañías farmacéuticas para localizar otros ensayos.

Selección de estudios: ensayos clínicos controlados aleatorizados (ECA) doble ciego que comparasen ácido ursodeoxicólico con placebo después de la cirugía bariátrica en pacientes con obesidad mórbida.

Evaluación de calidad y extracción de los datos: dos revisores de forma independiente utilizaron la escala de Jadad de calidad y extractaron los datos. Los desacuerdos se resolvieron por consenso.

Resultados de la revisión: se incluyeron cinco ECA ( $\mathrm{n}=521: 322$ ácido ursodeoxicólico y 199 con placebo). La media de edad de los participantes en los estudios incluidos varió desde 33,5 hasta 38 años. La proporción de mujeres osciló entre $56 \%$ a $82 \%$. Las dosis de ácido ursodeoxicólico oscilaron entre 300 mg y 1200 mg/día y la duración de los ensayos de tres a 24 meses. Todos los estudios fueron clasificados como de alta calidad aunque sólo dos tuvieron un seguimiento completo de todos los pacientes, el resto había perdido datos de entre $17,2 \%$ y $31,7 \%$ de los pacientes.Al realizar el análisis por intención de tratar, el uso de ácido ursodeoxicólico resultó en una reducción significativa del riesgo de formación de cálculos biliares después de la cirugía en comparación con el placebo (RR 0,43; IC95\% 0,22 a 0,83), aunque con una heterogeneidad significativa $(I 2=61,9 \%)$. El ensayo con la mayor pérdida en el seguimiento y peor cumplimiento de los pacientes con el tratamiento fue la causa de la mayor parte de la heterogeneidad observada, pero las conclusiones se mantuvieron cuando se excluyó este ensayo.Dos ensayos informaron efectos adversos. Uno reportó efectos secundarios leves a moderados y el otro que varios pacientes abandonaron a causa de vómitos o erupciones cutáneas, pero que los números fueron similares entre los grupos de tratamiento.

Conclusiones: el uso de ácido ursodeoxicólico en pacientes con la vesícula biliar alitiásica puede evitar la formación de cálculos biliares después de la cirugía bariátrica.

\section{Conclusiones generales}

La profilaxis con ácido ursodeoxicólico demostró ser efectiva en la reducción del riesgo de presentar colelitiasis durante el descenso rápido de peso. Esto se ve reflejado en los estudios expuestos. Ya sea en los grupos que se encontraban en un programa de descenso de peso como los pacientes sometidos a cirugía de restricción gástrica. Incluso se vieron efectos a más de un año de seguimiento. Además en uno de los estudios pudo apreciarse un efecto dosis respuesta, lo que sumado a la consistencia de los estudios refuerza mucho la plausibilidad del efecto preventivo del ácido ursodeoxicólico. De acuerdo en lo encontrado en estos trabajos, estaría recomendado el uso de ácido ursodeoxicólico en pacientes obesos que estén por comenzar una dieta de muy bajas calorías o por someterse a una cirugía de reducción gástrica.

Recibido 28/08/11 entregado 17/9/11

Ma. Soledad Mel. [ Alumna del Instituto Universitario del Hospital Italiano de Buenos Aires. maria.mel@ hospitalitaliano.org.ar ]

Referencias

1. Goroll A., Mulley A, "primary care medicine" office evaluation and Management of the adult patient, 6 th edition, pag. 553.

2. Afdhal NH. Epidemiology of and risk factors for gallstones. In: UpToDate, Basow, DS (Ed), UpToDate 18.3, Waltham, MA, 2010.

3. Jones D, Adair JD. Complications of bariatric surgery. In: UpToDate, Basow, DS (Ed), UpToDate 18.3, Waltham, MA, 2010. 